\title{
Correlação entre ruptura de ligamento cruzado cranial e lesão de menisco medial em cães
}

\author{
Correlation between cranial cruciate ligament rupture and meniscal tears in dogs
}

\author{
Ísis dos Santos Dal-Bó ${ }^{{ }^{*}}$ Cássio Ricardo Auada Ferrigno ${ }^{\mathrm{I}}$ Daniela Fabiana Izquierdo Caquías ${ }^{\mathrm{I}}$ \\ Marcos Ishimoto Della Nina ${ }^{I I}$ Márcio Poletto Ferreira ${ }^{\mathrm{I}}$ Adriana Valente de Figueiredo ${ }^{\mathrm{I}}$ \\ Renato Albuquerque de Oliveira Cavalcanti ${ }^{\mathrm{I}}$ Jaqueline França do Santos ${ }^{\mathrm{I}}$ \\ Vanessa Couto de Magalhães Ferraz ${ }^{\mathrm{I}}$
}

\section{RESUMO}

O presente trabalho correlacionou o tempo de ruptura do ligamento cruzado cranial (RLCCr) à lesão em menisco medial e ao tipo de lesão observada em cães tratados por estabilização dinâmica (osteotomias) ou estática (sutura fabelo-tibial). Este estudo retrospectivo avaliou os registros de 104 cães (117 joelhos). As osteotomias foram realizadas em 98 articulações, a sutura fabelo-tibial em 15 joelhos e, em 4 joelhos, foi realizada apenas artrotomia exploratória. A média de idade foi 4,5 anos, 41\% dos cães eram machos e 59\%, fêmeas e a média da massa corporal foi $32,7 \mathrm{~kg}$. Os tipos de lesão do menisco medial observados foram: tipo 1, 2, 3, 4, 6, 7 e associações dos tipos 1-4, 1-6 e 1-7. O tipo 1 foi o mais prevalente, sendo responsável por 32,5\% dos casos. Não foram encontradas correlações entre a presença ou não de lesão de menisco medial e o tempo de ocorrência da RLCCr e entre o tipo de lesão de menisco medial e o tempo de RLCCr.

Palavras-chave: TPLO, TTA, meniscectomia, joelho, canino.

\section{ABSTRACT}

The study correlated the time of the cranial cruciate ligament rupture (CCLR) with medial meniscal tears and the type of injury seen in dogs treated with dynamic stabilization (osteotomies) or static (fabellotibial suture). This retrospective study evaluated the records of 104 dogs (117 stifles). The osteotomies were performed in 98 stifles, fabellotibial suture in 15 stifles and exploratory arthrotomy was perform in 4 stifles. The average age was of 4.5 years, $41 \%$ of the dogs were male and 59\% female and the mean body mass was of $32.7 \mathrm{~kg}$. The medial meniscal tears observed were: type1, 2, 3, 4, 6, 7 and associations of types1-4, 1-6 and 1-7. Type 1 was the most prevalent, accounting for $32.5 \%$ of cases. No correlation was found between the presence or absence of medial meniscus injury and the time of occurrence of CCLR and between the type of medial meniscal tears and CCLR time.

Key words: TPLO, TTA, meniscectomy, stifle, dog.

\section{INTRODUÇÃO}

Osmeniscos são estruturas fibrocartilaginosas em formato de discos semilunares situados no interior do joelho. Estão ligados à tíbia através dos ligamentos meniscotibiais e entre eles pelo ligamento intermeniscal (CASE et al., 2008; FRANKLIN et al., 2010). O menisco medial é ligado à cápsula articular e ao ligamento colateral medial enquanto o lateral apresenta um anexo ligamentoso caudal ao fêmur e é relativamente pouco ligado à cápsula articular. $\mathrm{O}$ menisco medial, portanto, é mais firmemente ancorado à tíbia e frequentemente desliza cranialmente ao fêmur durante a translação tibial em joelhos instáveis, o que o torna mais propenso às lesões. O menisco lateral apresenta maior liberdade e, por isso, mantém posição neutra durante os episódios de subluxação fêmorotibial (FRANKLIN et al., 2010; POZZI \& COOK, 2010a), sendo a lesão em menisco lateral incomum (NECAS \& ZATLOUKAL, 2002).

As lesões em menisco medial primárias são raras, entretanto, lesões secundárias à ruptura do ligamento cruzado cranial (RLCCr), podem ocorrer em 40 a $60 \%$ dos cães, sendo mais frequentes em joelhos cronicamente instáveis (AUSTIN et al., 2007; FRANKLIN et al., 2010). Estas são classificadas em: tipo 1 - eversão do corno caudal; tipo 2 - longitudinal; tipo 3 - longitudinal múltipla; tipo 4 - fibrilação/ degeneração da superfície; tipo 5 - axial; tipo 6 alça de balde; e tipo 7 - transversal, sendo os tipos 1, 2 e 6 mais frequentes (BENNETT \& MAY, 1991; SCHULZ, 2007).

'Laboratório de Ortopedia e Traumatologia Comparada do Departamento de Cirurgia (LOTC), Faculdade de Medicina Veterinária e Zootecnia (FMVZ), Universidade de São Paulo (USP), 05508-270, São Paulo, SP, Brasil. E-mail: idb_vet@yahoo.com.br. *Autor para correspondência.

"Médico Veterinário autônomo, São Paulo, SP, Brasil. 
O presente trabalho correlacionou o tempo de ruptura de ligamento cruzado cranial à ruptura de menisco medial, bem como ao tipo de lesão meniscal, em cães tratados com estabilização dinâmica ou estática.

\section{MATERIAL E MÉTODO}

Este estudo retrospectivo avaliou o registro de 104 cães (117 joelhos) atendidos no Laboratório de Ortopedia e Traumatologia Comparada do Departamento de Cirurgia da Faculdade de Medicina Veterinária e Zootecnia da Universidade de São Paulo (LOTC-FMVZ/USP) que haviam sido submetidos às osteotomias corretivas (TTA - tibial tuberosity advancement, TPLO - tibial plateau leveling osteotomy e CWO - closing wedge osteotomy) e estabilização extracapsular (sutura fabelo-tibial) para tratamento de RLCCr durante o período de janeiro de 2006 a novembro de 2012.

Foram realizados testes de gaveta e compressão tibial em todos os animais com propósito de identificar instabilidade articular no joelho, além da flexão e extensão, com intuito de identificar ruído meniscal audível ou deslocamento meniscal palpável, principalmente ao final do movimento de flexão, conforme descrito por SCHULZ (2007) e CASE et al. (2008). Além disso, os pacientes foram radiografados sob sedação (acepromazina $0,05 \mathrm{mgkg}^{-1}$ e butorfanol $0,1 \mathrm{mgkg}^{-1}$, por via intramuscular), na projeção mediolateral, tendo suas articulações fêmoro-tíbiopatelares a $135^{\circ}$ e tíbio-társica a $90^{\circ}$ de flexão. A seguir, ambas foram flexionadas a $90^{\circ}$ e novamente radiografadas na projeção mediolateral, conforme recomendado por AULAKH et al. (2011). Também foram realizadas radiografias da articulação fêmorotíbio-patelar na projeção craniocaudal. O estudo radiológico foi desenvolvido com o intuito de realizar o planejamento cirúrgico por meio da mensuração do ângulo do platô tibial e escolha da osteotomia corretiva a ser empregada, bem como para diagnóstico ou avaliação da progressão de doença articular degenerativa préexistente nos joelhos. Os cães que apresentaram ângulo do platô tibial de até $25^{\circ}$ foram submetidos à TTA e os que mediram mais que $25^{\circ}$ foram selecionados para TPLO ou CWO. A sutura fabelo-tibial foi indicada para os pacientes com ângulo do platô tibial em torno de $25^{\circ}$ e massa corporal de até $10 \mathrm{~kg}$.

A avaliação da integridade dos meniscos foi realizada por meio de miniartrotomia fêmorotíbio-patelar medial, feita imediatamente antes da osteotomia ou sutura fabelo-tibial. O menisco medial foi visibilizado com o auxílio de afastadores de Hohmann e Gelpi, e probe artroscópica, o que facilitou sua exposição e manipulação. As lesões meniscais foram classificadas segundo BENNETT \& MAY (1991) e SCHULZ (2007), sendo que alguns pacientes apresentavam mais de uma lesão. Estas foram tratadas por meio de meniscectomia parcial. Nos animais em que não foram encontradas alterações meniscais, foi realizada liberação do menisco medial.

Determinou-se o tempo decorrido entre o surgimento dos sinais clínicos até o procedimento cirúrgico e, a partir desses dados, foi calculado o tempo da ruptura do ligamento cruzado cranial. Este foi correlacionado à presença ou ausência de lesão de menisco medial e ao tipo de lesão.

A análise estatística foi realizada por meio do teste de Kruskal-Wallis, comparando-se a presença ou não de lesão de menisco medial com o tempo de ruptura do ligamento cruzado cranial e os tipos mais frequentes de lesão com o tempo transcorrido da ruptura ligamentar, até a intervenção cirúrgica de estabilização articular.

\section{RESULTADOS}

Foram avaliados 104 cães (117 joelhos) que apresentavam RLCCr. Destes, 97 joelhos foram diagnosticados com ruptura de menisco medial e os outros 20 apresentaram meniscos íntegros. As osteotomias corretivas foram realizadas em 98 articulações: TTA $n=51$ (43,6\%), TPLO n=35 (29,9\%) e CWO $n=12$ (10,2\%). A sutura fabelo-tibial foi a técnica escolhida para 15 joelhos (12,8\%) e, em 4 (3,4\%), foi realizada apenas artrotomia exploratória para avaliação do menisco medial. A RLCCr bilateral foi observada em 13 pacientes, no entanto, os procedimentos para estabilização articular não foram realizados simultaneamente.

As raças mais prevalentes foram Labrador Retriever ( $n=23)$, Rottweiler $(n=17)$, American Pit Bull Terrier ( $n=13)$, sem raça definida (SRD) $(n=12)$, Boxer $(n=9)$, Chow-chow $(n=6)$ e Poodle $(n=5)$. A média de idade foi de 4,5 anos, variando de 1,5 a 12 anos, sendo que 26,5\% dos animais apresentaram até 2 anos, 33,3\% entre 2 a 5 anos e 40,1\%, mais de 5 anos. Quanto ao sexo, 41\% eram machos e 59\%, fêmeas. A massa corpórea variou entre 4 e $78 \mathrm{~kg}$ (média de 32,7kg), 63,2\% dos animais apresentaram mais de $30 \mathrm{~kg}$ e $36,7 \%$, até $30 \mathrm{~kg}$.

Os tipos de lesão do menisco medial encontrados neste estudo foram 1, 2, 3, 4, 6, 7 e associações dos tipos 1-4, 1-6 e 1-7. O tipo 1 foi o mais prevalente, sendo observado em 32,5\% dos casos, seguido pela associação entre os tipos 1 e 6 
$(17,0 \%)$ e pelo tipo $6(16,2 \%)$. Os dados referentes ao tipo de lesão de menisco medial estão descritos na tabela 1. Os pacientes foram divididos em grupos, de acordo com o tempo de RLCCr em até 30, 60, 90, 120, 150180 e mais de 180 dias, sendo o último intervalo o mais prevalente nos pacientes (34,0\%). Esses dados se encontram na tabela 2.

Foi realizada análise estatística correlacionando os grupos sem lesão, lesão do tipo 1 , tipo 6 e com lesões do tipo 1 e 6 concomitantes com o tempo transcorrido entre a ruptura e o tratamento cirúrgico. Nessa análise, estas lesões acometeram a maior parte dos pacientes avaliados no estudo e isto possibilitou avaliação estatística em grupos mais homogêneos, excluindo assim grupos de lesões em que o número de pacientes foi muito discrepante.

A análise estatística foi realizada por meio dos testes Kruskal-Wallis. Não foram encontradas correlações entre a presença ou não de lesão de menisco medial e o tempo de ocorrência da RLCCr. No que tange à avaliação entre o tipo de lesão de menisco medial e o tempo de RLCCr, também não foram considerados significantes os resultados encontrados nos testes estatísticos realizados.

\section{DISCUSSÃO}

O diagnóstico definitivo da lesão de menisco é realizado através de artrotomia ou artroscopia. Neste estudo, a miniartrotomia medial se mostrou eficaz na avaliação do menisco medial, entretanto COOK \& POZZI (2010) afirmaram que há a necessidade da utilização de afastadores, além da avaliação do joelho em vários graus de extensão

Tabela 1 -Número de animais de acordo com o tipo de lesão de menisco medial, relacionado à cronicidade da ruptura de ligamento cruzado cranial (RLCCr).

\begin{tabular}{llll}
\hline $\begin{array}{l}\text { Tipo de lesão de } \\
\text { menisco medial }\end{array}$ & $\begin{array}{l}\text { Número de } \\
\text { animais }\end{array}$ & $\%$ & $\begin{array}{l}\text { Cronicidade da } \\
\text { RLCCr (dias) }\end{array}$ \\
\hline Sem lesão & 20 & 17 & 219,7 \\
Tipo 1 & 38 & 32,5 & 173 \\
Tipo 2 & 5 & 4,3 & 158,4 \\
Tipo 3 & 1 & 0,8 & 151 \\
Tipo 4 & 9 & 7,7 & 132,4 \\
Tipo 6 & 19 & 16,2 & 198,5 \\
Tipo 7 & 1 & 0,8 & 27 \\
$\begin{array}{l}\text { Associação dos } \\
\text { tipos 1 e 4 }\end{array}$ & 2 & 1,7 & 138,5 \\
$\begin{array}{l}\text { Associação dos } \\
\text { tipos 1 e 6 }\end{array}$ & 20 & 17 & 192 \\
$\begin{array}{l}\text { Associação dos } \\
\text { tipos 1 e 7 }\end{array}$ & 2 & 1,7 & 239,5 \\
Total & 117 & 100 & -- \\
\hline
\end{tabular}

Tabela 2 - Cronicidade da ruptura de ligamento cruzado cranial (RLCCr) dividida em grupos.

\begin{tabular}{lcl}
\hline Cronicidade da RLCCr & Número de animais & $\%$ \\
\hline Até 30 dias & 8 & 7 \\
Até 60 dias & 15 & 12,8 \\
Até 90 dias & 23 & 19,7 \\
Até 120 dias & 14 & 12 \\
Até 150 dias & 9 & 7,7 \\
Até 180 dias & 8 & 6,8 \\
Mais que 180 dias & 40 & 34 \\
Total & 117 & 100 \\
\hline
\end{tabular}

e flexão, com estresse varo e valgo e em rotação interna e externa, visando adequada exposição das estruturas. Tais manobras não são necessárias nos procedimentos artroscópicos, considerados padrãoouro para diagnóstico e tratamento das lesões de menisco, os quais permitem manipulação tecidual minimamente invasiva e visibilização completa com magnificação da imagem (COOK \& POZZI, 2010).

O tipo de lesão de menisco medial mais prevalente foi o tipo 1 - eversão do polo caudal $(n=38)$, contrapondo os resultados de CASE et al. (2008), em que a lesão mais prevalente foi a tipo 6 (ruptura em alça de balde) em 26 cães e FITZPATRICK et al. (2010), que observaram em 1000 cães maior prevalência da associação dos tipos 1 e 6, após a realização de TPLO. Contudo, os dados obtidos corroboram os encontrados por FERRIGNO et al. (2012), que observaram, em 82 pacientes, a ocorrência de lesão tipo 1 em 33 joelhos, sendo esta considerada como a mais frequente.

Para tratamento das lesões meniscais, optou-se pela meniscectomia parcial, através de artrotomia medial, assim como relatado por STEINBERG et al. (2011) e WOLF et al. (2012). A perda ou a remoção de tecido meniscal diminui o suporte de carga, absorção de choque, congruência, estabilidade articular, nutrição tecidual e funções condroprotetoras dos meniscos, fazendo com que a meniscectomia esteja associada ao desenvolvimento de doença articular degenerativa e condromalácia em cães (MARTIG et al., 2006; COOK \& POZZI, 2010). No presente estudo, contudo, não foi possível avaliar o desenvolvimento dessas alterações após a meniscectomia, uma vez que a maioria dos pacientes apresentava, no momento do primeiro atendimento clínico, algum grau de osteoartrose.

O desenvolvimento da doença articular degenerativa, conforme referido por POZZI et al. (2010), é desencadeado pela instabilidade articular secundária à insuficiência do ligamento cruzado cranial, contudo, a liberação do menisco medial pode contribuir para progressão da degeneração da 
cartilagem, mesmo após estabilização cirúrgica. Nos pacientes em que não foi identificada lesão de menisco, entretanto, foi realizada liberação do menisco medial, ou seja, a transecção radial, liberando o polo caudal da exposição às cargas anormais durante o apoio do membro. O objetivo desse procedimento é eliminar o impacto no menisco entre o côndilo femoral e o platô tibial, fazendo com que o menisco medial se torne menos fixo, assim como o lateral, e diminuindo a probabilidade de futuras lesões após a estabilização articular (POZZI \& COOK, 2010b). Essa técnica é defendida por muitos autores, contudo, ainda é recente e não há comprovação se está relacionada ao desenvolvimento ou à progressão da osteoartrose (POZZI et al., 2008). Nenhum dos pacientes submetidos à liberação de menisco apresentou piora no quadro clínico. Além disso, a realização da liberação de menisco reduz a possibilidade de segunda intervenção cirúrgica após a osteotomia, uma vez que 5,2\% dos cães podem desenvolver lesões meniscais após TTA (STEINBERG et al., 2011) e 6,3\% após TPLO (THIEMAN et al., 2006), quando a liberação do menisco medial não é realizada.

Foram encontradas lesões associadas de menisco dos tipos 1 e 4, 1 e 6 e 1 e 7 em 24 pacientes, em contraste com o observado por THIEMAN et al. (2006), que, após avaliar 254 joelhos, encontrou apenas três pacientes com lesões meniscais combinadas. Entretanto, a presença de lesões concomitantes também foi observada por FITZPATRICK et al. (2010).

Nas lesões de menisco medial associadas à RLCCr em cães, a imediata correção cirúrgica da lesão de ligamento é recomendada com o intuito de prevenir a progressão da doença articular degenerativa, especialmente em cães com massa corporal acima de 15kg (ERTELT \& FEHR, 2009). Entretanto, os proprietários dos pacientes incluídos nesse trabalho não consideraram a claudicação como urgência para procura de atendimento veterinário, pois houve animais que foram submetidos à cirurgia mais de três anos após o aparecimento dos sinais clínicos.

\section{CONCLUSÃO}

Nos pacientes avaliados neste estudo, não houve correlação entre o tempo de ruptura do ligamento cruzado cranial e o tipo de lesão em menisco medial ou desta com a presença de lesão em menisco.

\section{COMTÊ DE ÉTICA E BIOSSEGURANÇA}

Venho através desta, esclarecer que este trabalho não foi submetido à avaliação pelo Comitê de Ética e Biossegurança, por se tratar de um estudo retrospectivo, cujos dados foram obtidos dos prontuários de pacientes que foram atendidos na rotina clínico-cirúrgica do Laboratório de Ortopedia e Traumatologia Comparada do Departamento de Cirurgia (LOTC), Faculdade de Medicina Veterinária e Zootecnia (FMVZ), Universidade de São Paulo (USP). Afirmo que os animais não foram submetidos a procedimentos desnecessários e que todas as medidas (incluindo anestesia e analgesia adequadas para cada caso) para evitar a dor e o sofrimento foram tomadas. Dessa forma, assumo toda e qualquer responsabilidade sobre as informações aqui contidas, representando também os outros autores do artigo.

\section{REFERÊNCIAS}

AULAKH, K.S. et al. Effect of stifle angle on the magnitude of the tibial plateau angle measurement in dogs with intact and transected cranial cruciate ligament. Veterinary and Comparative Orthopaedics and Traumatology, v.22, p.272-278, 2011. Disponível em: <http://www.schattauer.de/en/magazine/ subject-areas/journals-a-z/vcot/contents/archive-2003/issue/1424/ manuscript/15977/show.html>. Acesso em: 14 jul. 2013.

AUSTIN, B. et al. Evaluation of the three approaches to meniscal release. Veterinary and Comparative Orthopaedics and Traumatology, v.2, p.92-97, 2007. Disponível em: <http:// www.schattauer.de/en/magazine/subject-areas/journals-a-z/vcot/ contents/archive-2003/issue/135.html>. Acesso em: 13 maio 2013.

BENNETT, D.; MAY C. Meniscal damage associated with cruciate disease in the dog. Journal of Small Animal Practice, v.32, n.3, p.111-117, 1991.

CASE, J.B. et al. Meniscal injury following initial cranial cruciate ligament stabilization surgery in 26 dogs. Veterinary and Comparative Orthopaedics and Traumatology, v.4, p.365-367, 2008. Disponível em: <http://www.schattauer.de/en/magazine/ subject-areas/journals-a-z/vcot/contents/archive-2003/issue/132. html>. Acesso em: 13 maio 2013.

COOK, J.L.; POZZI A. Surgical treatment of concurrent meniscal injury. In: MUIR P. Advances in the canine cranial cruciate ligament. Ames: Wiley-Blackwell, 2010. Cap.31, p.217-222.

ERTELT, J.; FEHR M. Cranial cruciate ligament repair in dogs with and without meniscal lesions treated by different minimally invasive methods. Veterinary and Comparative Orthopaedics and Traumatology, v.1, p.21-26, 2009. Disponível em: <http:// www.schattauer.de/en/magazine/subject-areas/journals-a-z/vcot/ contents/archive-2003/issue/126.html>. Acesso em: 13 maio 2013.

FERRIGNO, C.R.A. et al. Ruptura de menisco associada à ruptura de ligamento cruzado em cães. Brazilian Journal of Veterinary Research and Animal Science, v.49, n.4, p.301-306, 2012. Disponível em: <http://www.revistas.usp.br/bjvras/article/ view/51717>. Acesso em: 13 maio 2013.

FITZPATRICK, N. et al. Predictive variables for complications after TPLO with stifle inspection by arthrotomy in 1000 consecutive dogs. Veterinary Surgery, v.39, p.460-474, 2010. Disponível em: <http://onlinelibrary.wiley.com/doi/10.1111/ j.1532-950X.2010.00663.x/pdf>. Acesso em: 13 maio 2013.

FRANKLIN, S.P. et al. Meniscal injury in dogs with cranial cruciate ligament rupture. Compendium: Continuing Education for Veterinarians. 2010. Disponível em: <http://mobile.vetlearn. 
com/Media/images/pdf/2010/PV/PV1010_franklin_CE.pdf>. Acesso em 06 out. 2012.

MARTIG, S. et al. Low-field MRI and arthroscopy of meniscal lesions in ten dogs with experimental induced cranial cruciate ligament insuficiency. Veterinary Radiology and Ultrasound. v.47, n.6, p.515-522, 2006. Disponível em: <http://onlinelibrary. wiley.com/doi/10.1111/j.1740-8261.2006.00179.x/pdf>. Acesso em: 13 maio 2013.

NECAS, A.; ZATLOUKAL, J. Factors related to the risk of meniscal injury in dogs with cranial cruciate ligament rupture. Acta Veterinaria Brno, v.71, p.77-84, 2002.

POZZI, A.; COOK, J.L. Meniscal structure and function. In: MUIR P. Advances in the canine cranial cruciate ligament. Ames: Wiley-Balckwell, 2010a. Cap.4, p.29-35.

POZZI, A.; COOK, J.L. Meniscal release. In: MUIR P. Advances in the canine cranial cruciate ligament. Ames: Wiley-Balckwell, 2010b. Cap.32, p.223-228.

POZZI, A. et al. Pressure distributions on the medial tibial plateau after medial meniscal surgery and tibial plateau levelling osteotomy in dogs. Veterinary Comparative Orthopaedics and Traumatology, v.1, p.8-14, 2008. Disponível em: <http:// www.schattauer.de/en/magazine/subject-areas/journals-a-z/ vcot/contents/archive-2003/issue/129.html>. Acesso em: 13 maio 2013.

POZZI, A. et al. Effect of transection of the caudal menisco-tibial ligament on medial femorotibial contact mechanics. Veterinary Surgery, v.39, p.489-495, 2010. Disponível em: <http:// onlinelibrary.wiley.com/doi/10.1111/j.1532-950X.2010.00662.x/ pdf $>$. Acesso em: 15 jul. 2013

SCHULZ, K. Diseases of the joint. In: FOSSUM, T.W. (Ed). Small animal surgery. 3.ed. St Louis: Mosby, 2007. Cap.33, p.1285-1289.

STEINBERG, E.J. et al. Tibial tuberosity advancement for treatment of CrCL injury: complications and owner satisfaction. American Animal Hospital Association, v.47, n.4, p.250-257, 2011.

THIEMAN, K.M. et al. Effect of meniscal release on rate of subsequent meniscal tears and owner-assessed outcome in dogs with cruciate disease treated with tibial plateau leveling osteotomy. Veterinary Surgery, v.35, p.705-710, 2006. Disponível em: <http:// onlinelibrary.wiley.com/doi/10.1111/j.1532-950X.2006.00214.x/ pdf>. Acesso em: 13 maio 2013.

WOLF, R.E. et al. Surgical and postoperative complications associated with tibial tuberosity advancement for cranial cruciate ligament rupture in dogs: 485 cases (2007-2009). Journal of the American Veterinary Medical Association, v.240, p.481-1487, 2012. 